JSPS Grants-in-Aid for Creative Scientific Research

Understanding Inflation Dynamics of the J apanese Economy Working Paper Series No.16

\title{
Unobserved Heterogeneity in Price-Setting Behavior: a Duration Analysis Approach
}

\author{
Takayasu Matsuoka
}

October 24, 2007

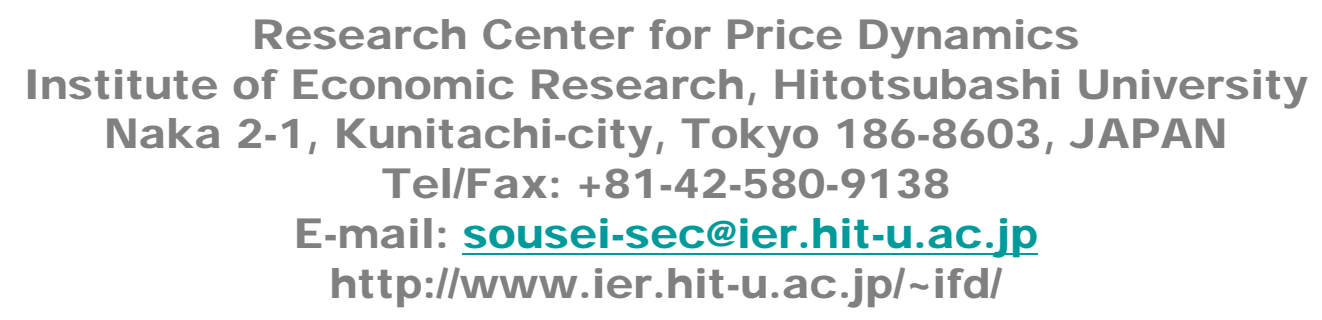




\title{
Unobserved Heterogeneity in Price-Setting Behavior: a Duration Analysis Approach
}

\author{
Takayasu Matsuoka* \\ Graduate School of Economics, Osaka University \\ JSPS Research Fellow
}

October 2007

\begin{abstract}
There is strong empirical evidence that the degree of price stickiness differs across commodity items, and that the nonparametric hazard function of price changes is downward-sloping with some spikes. We introduce item-specific heterogeneity into the standard single-sector model of Calvo (1983) and estimate a hazard function of price adjustment, by applying duration analysis. We present the appropriate form of heterogeneity for the data structure, and show that the decreasing (population) hazard function is well described. In the presence of item-specific heterogeneity, the probability that prices remain unchanged is predicted to be higher than in the single-sector model.
\end{abstract}

JEL classification codes: D40, E31, C41

Key words: Sticky prices, Hazard function, Heterogeneity

\footnotetext{
* I would like to thank Kanemi Ban, Yukinobu Kitamura, Tsutomu Watanabe, Kazuo Ogawa, Charles Yuji Horioka, Yuichi Fukuta, Tomoyoshi Yabu, Teruyoshi Kobayashi, Hiroki Arato and seminar participants at Osaka University, Summer Workshop on Economic Theory, and the Second Conference on Applied Econometrics for helpful comments. Any remaining errors are my own. This study is financially supported by the research fellowships of the Japan Society for the Promotion of Science for young scientists. Address for correspondence: 1-7 Machikaneyama, Toyonaka, Osaka 560-0043, Japan. Tel.: +81 905009 4141. E-mail: fgp019mt@mail2.econ.osaka-u.ac.jp.
} 


\section{Introduction}

Previous studies (Bils and Klenow, 2004; Dhyne et al., 2005; Saita et al., 2006) have shown that the degree of price stickiness differs across commodity items. The time-dependent pricing model (Calvo, 1983), in which one single parameter represents price stickiness, cannot reproduce the strong empirical evidence in such a way that the nonparametric hazard function of price changes is decreasing (Álvarez, Burriel and Hernando, 2005). Each item has specific factors related to its survival experience. These specific factors, whether observable or not, change the shape of the (individual) hazard function. If the variability in hazard is not fully captured by covariates, it is necessary to model unobserved heterogeneity.

However, in many empirical works on price-setting behavior unobserved heterogeneity is left unspecified. Therefore, we introduce item-specific heterogeneity into Calvo's (1983) standard single-sector model, and estimate the hazard function of price adjustment, by applying duration analysis. This paper provides the appropriate form of heterogeneity for the data structure, and shows that the decreasing (population) hazard is well described by modeling item-specific heterogeneity.

Recent papers that analyze monetary shock by the calibrated dynamic general equilibrium model show that the degree of monetary non-neutrality implied by a multi-sector model is larger than that implied by its single-sector counterpart calibrated to the mean frequency of price change. The response to monetary shock is much larger in a sticky sector. These findings are consistent with our result that the existence of heterogeneity implies the speed of price adjustment slows, because over a period of time the effect of price spells with long duration gradually dominates.

The paper is organized as follows. Section 2 summarizes our approach. Section 3 presents the model with unobserved heterogeneity that is shared across price spells within an item. This is called the shared frailty model. Section 4 discusses the results. 


\section{Duration approach to the problem of price stickiness}

Following Aucremanne and Dhyne (2004), the methods used in this field of research are the frequency approach and the duration approach. This paper employs the latter. The frequency approach first calculates the monthly frequency of price changes by items, which is equal to the total number of price changes divided by the total number of observed prices. It then aggregates the frequencies weighted by CPI weight to obtain the mean frequency. The expected value of waiting time is a reciprocal of the frequency of price change. Therefore, the mean frequency implies that the expected length of price spell is

$$
\frac{1}{\delta}=-\frac{1}{\ln (1-\lambda)}
$$

where $\lambda$ is monthly frequency and $\delta$ is implied instantaneous frequency ${ }^{1}$. The median of price-change frequency is the middle value of weighted frequency which implies the median duration. Bils and Klenow (2004) use the frequency approach by first computing the frequency of price changes, and then inferring the implied average duration of a price spell for each product category in the U.S. consumer price index.

The duration approach first specifies the functional form of the hazard function. The price-setting behavior described by the Calvo model corresponds to the exponential model with constant hazard rate. Calvo (1983) assumes the probability density function of a price spell with duration $t$ as follows:

$$
\delta \cdot e^{-\delta t}, \quad \delta>0
$$

This function is divided in two parts. The first, $\delta$, is the hazard function in the Calvo

\footnotetext{
${ }^{1}$ Monthly frequency and instantaneous frequency satisfy the equality $\lambda=1-e^{-\delta}$. If we subdivide one month into $\mathrm{n}$ equal intervals and assume all firms change their price with probability $\delta \Delta t$ during the period $(t, t+\Delta t)$, the probability that price change does not occur for one month is $(1-\delta \Delta t)^{n}$. Therefore the monthly probability of price change is $\lambda=1-(1-\delta \Delta t)^{n}$. Letting $\Delta t \rightarrow 0$, we obtain $\lambda=1-e^{-\delta}$, or equivalently, $\delta=-\ln (1-\lambda)$.
} 
model, which is the instantaneous price-change probability. The second, $e^{-\delta t}$, is the survivor function, i.e., the probability that a price has not been changed until t. We construct the likelihood function according to this functional form, and maximize it using all the data to obtain the maximum likelihood estimators, which summarize the shape of hazard function and survivor function. The median duration is the elapsed time, which satisfies the condition that the survival probability is 0.5: i.e., the proportion of unchanged price spells is only 50 percent.

One advantage of this approach is that it clearly evaluates the pattern of price adjustment. As we show later, the nonparametric hazard rate is significantly higher at 12, 24, and 36 month suggesting that price changes tend to occur annually. We can introduce various types of heterogeneity. Álvarez, Burriel, and Hernando (2005) use finite mixture models, which presuppose that the population consists of homogeneous subpopulations. They specify the hazard function for each subpopulation, and show that the mixture of hazard functions varies according to how they specify functional forms, and how many subpopulations the model contains. They document that it is optimal to estimate a model composed of 3 groups with a different but constant hazard rate, plus 1 group with a positive hazard rate at every 12 months. In our shared frailty model, we assume a priori that the population consists of heterogeneous items, which is supported by the result that the degree of price stickiness differs across items. Formally, the difference between the finite mixture model and our shared frailty model is that the former is a fixed-effects model with random groups, whereas the latter is a random-effects model with known groups. ${ }^{2}$

\footnotetext{
2 See Mosler (2003) and Cameron and Trivedi (2005) for further discussion.
} 


\section{Shared frailty model for price-setting behaviors}

In this analysis, we employ a sample of the retail prices underlying the computation of the Japanese consumer price index. These prices are collected on a monthly basis by the Statistic Bureau, Ministry of Internal Affairs and Communication, and appear in the Monthly Report on the Retail Price Survey. Prices are reported for each city with a prefectural government, and for cities with a population of 150,000 or more. The analysis covers the period from January 2000 to December 2005. Our raw data set considers 498 items, covering $68 \%$ of the Japanese CPI in 2000, and consisting of 2,063,148 price records.

Our data is a Japanese counterpart of previous studies following Bils and Klenow (2004). These studies use outlet-level price data; however, we use the average of prices quoted at each outlet. Since we cannot access the price data of each outlet where the price report was conducted, our data set provides the best available information in Japan for measuring the degree of price stickiness. Saita et al. (2006) also use the source. As they note, the frequency of price change may have an upper bias when we use average prices, because we count the number of price changes even if some outlet-level prices do not change.

We assume that price change does not occur more than twice because our data is monthly data, to enable us to observe the price of each category only once a month. This is a limitation of our analysis, and of all previous studies using monthly data.

Table 1 describes our data set. In our retail price data, several price spells are observed per item. We thus call them multiple-spell data. When we compute the duration of price spells directly, we need to trim the original dataset. First, we discard all left-censored spells. The duration of price spells quoted before the beginning of our observation period cannot be calculated, because we do not know the starting time of price spells. This is the problem of left-censoring (Amemiya, 1984). Since our data is multiple-spell data and the observation period is sufficiently long, this exclusion does not create serious problems. Second, we remove price spells that end with an item substitution. This is also negligible, because these spells constitute a small fraction of the total of price spells.

To analyze item-specific effects, we must assume that the heterogeneities are not 
specific to a price spell, but are shared within each item. Therefore, the multiple-spell data lead to a hierarchical structure. Specifically, let $v_{i}(i=1, \cdots, G)$ be independently and identically distributed random variables with a common distribution. We assume the hazard function for the $i$ th subject in the $j$ th group given the $j$ th heterogeneity is

$$
\lambda_{i j}(t)=v_{i} \cdot \lambda\left(t \mid X_{i j}\right)
$$

This implies that the cumulative hazard function for the same subject conditional on the $j$ th heterogeneity is

$$
\Lambda_{i j}(t) \equiv \int_{0}^{t} \lambda_{i j}(s) d s=v_{i} \int_{o}^{t} \lambda\left(s \mid X_{i j}\right) d s=v_{i} \cdot \Lambda\left(t \mid X_{i j}\right)
$$

Using the identity $\Lambda(t)=-\ln \{S(t)\}$, we obtain the conditional survivor function

$$
S_{i j}(t)=\left[S\left(t \mid X_{i j}\right)\right]^{v_{i}}
$$

For the $i$ th subject in the $j$ th group, the shared frailty model treats the hazard as equation (3). We assume that the shared frailties are i.i.d. samples from a Gamma distribution. Since the scale parameter of the distribution is unidentifiable, the mean and variance are normalized to set $E[v]=1$ and $V[v]=\delta$, respectively. ${ }^{3}$ The density therefore becomes

$$
g(v)=\frac{v^{(1 / \delta-1)} \exp (-v / \delta)}{\Gamma(1 / \delta) \delta^{1 / \delta}}, \quad v>0
$$

The joint survivor function for the $i$ th group is given by

\footnotetext{
${ }^{3}$ The gamma distribution $\Gamma(k, \delta)$ has $E[v]=k \delta, V[v]=k \delta^{2}$. Setting $k=1 / \delta$, we obtain the normalized parameter values.
} 


$$
\begin{aligned}
S\left(t_{i 1}, \cdots, t_{i i_{i}}\right) & =\operatorname{Pr}\left[T_{i 1}>t_{i 1}, \cdots, T_{i n_{i}}>t_{i n_{i}}\right] \\
& =L P\left[\sum_{j=1}^{n_{i}} \Lambda\left(t_{i j} \mid X_{i j}\right)\right] \\
& =\left[1+\delta \sum_{j=1}^{n_{i}} \Lambda\left(t_{i j} \mid X_{i j}\right)\right]^{-1 / \delta}
\end{aligned}
$$

Here, $L P(x)=E_{v}[\exp (-v x)]$ is the Laplace transform of the frailty $v$. See Appendix A for the derivation in detail. The log-likelihood contribution of the $i$ th group is

$$
L_{i}(\delta, \lambda, \beta)=\left[\prod_{j=1}^{n_{i}} \lambda\left(t_{i j} \mid X_{i j}\right)^{d_{i j}}\right] \frac{\Gamma\left(1 / \delta+D_{i}\right)}{\Gamma(1 / \delta)} \delta^{D_{i}}\left[1+\delta \sum_{j=1}^{n_{i}} \Lambda\left(t_{i j} \mid X_{i j}\right)\right]^{-\left(1 / \delta+D_{i}\right)},
$$

where $d_{i j}$ is the indicator of the price change and $D_{i}=\sum_{j=1}^{n_{i}} d_{i j}$ is the number of price changes in the $i$ th group. We discuss this in detail in Appendix B. We then obtain the full log-likelihood

$$
l(\delta, \lambda, \beta)=\log \prod_{i=1}^{G} L_{i}(\delta, \lambda, \beta)
$$

We specify that the baseline hazard function is constant over time, i.e., the baseline hazard function and the cumulative hazard function become $\lambda\left(t \mid X_{i j}\right)=\lambda$ and $\Lambda\left(t \mid X_{i j}\right)=\lambda t$, respectively. Referring to equation (3), even though the baseline hazard is constant and the same across the items, the individual hazard functions $\lambda_{i j}(t)$ may differ because of frailty $v_{i}$. This exponential model with Gamma shared frailty is the natural extension of Calvo model, and allows price stickiness to vary across items. 


\section{The Estimation Results}

Since we specify the baseline hazard function, the maximum likelihood estimators are obtained by maximizing equation (6) using the Newton-Raphson method. ${ }^{4}$

Figure 1 shows the predicted hazard from the parametric shared frailty model and nonparametric hazard function, i.e., the Kaplan-Meier product limit estimators of hazard rate. The shared frailty model reproduces the decreasing hazard function and describes the shape of the nonparametric hazard function fairly well, except for short-run prediction. If we ignore unobserved heterogeneity, the hazard rate is constant at 0.365 , as shown in Table 2. This means that Calvo model overestimates the hazard rate for a long-term spell.

One reason is that, in the Calvo model, the hazard rate of price changes is common to all items, because it assumes that economy consists of homogeneous firms, which adjust their price randomly, but share a common probability of price change. This assumption of homogeneity cannot be supported empirically. The estimated frailty variance $\hat{\delta}$ is 1.149. Examining the likelihood-ratio test of $H_{0}: \delta=0$, the null hypothesis is soundly rejected at the $1 \%$ level of significance. Therefore, we conclude that the prediction errors are caused by item-specific heterogeneity.

Figure 2 shows that the survival probabilities of the Calvo model are significantly smaller than those of the shared frailty model. The median duration from the shared frailty model is slightly longer than that from the Calvo model. The predicted median duration is 2.87 months, which is about 1.5 times longer than the median duration implied by the price-change frequency of the single-sector model. The significant difference lies in the predictions at longer durations. The smaller probabilities in Calvo model arise because it ignores the effect of sticky items, which gradually dominate over time.

The implication of our findings relates to an import matter for monetary economics, since the dynamics of monetary economies depend to some extent on how to deal with sectors with a lower frequency of price change.

\footnotetext{
4 If we fit a Cox model with shared frailty in which the baseline hazard function is not specified, the estimates are obtained by using an EM algorithm. See Klein and Moeschberger (1997) and Yu (2006) for further discussion. Nakamura and Steinsson (2006b) use Cox proportional hazard model with covariates (seasonal dummies) and Gamma frailty and analyze the baseline hazard function by sector.
} 
Recent papers that analyze monetary shock using the dynamic stochastic general equilibrium model show that the degree of monetary shock implied by a multi-sector model is larger and more persistent than that implied by a single-sector model calibrated to the mean frequency of price change (Carvalho, 2006; Nakamura and Steinsson, 2006a). Carvalho (2006) introduces heterogeneity into Calvo’s (1983) model and concludes that to better approximate a single-sector model requires a much lower frequency of price changes than a multi-sector model. Our main finding can be restated as follows: the existence of heterogeneity implies that the speed of price adjustment slows down as time elapses. To approximate the survivor function of the shared frailty model, it is necessary to use a lower value for the hazard rate in the Calvo model. This conclusion is consistent with Carvalho (2006). 


\section{Conclusion}

This paper analyzes the heterogeneity in price-setting behavior using duration analysis. We introduce item-specific heterogeneity into the single-sector model, and show that the shared frailty model reproduces the decreasing hazard function, and describes the shape of the nonparametric hazard function fairly well.

In the Calvo (1983) model, the hazard rate of price changes is common to all items, because it assumes that the economy consists of homogeneous firms. We establish that this assumption of homogeneity cannot be supported empirically. We examine the likelihood-ratio test of the null hypothesis that the frailty variance is equal to zero. The hypothesis is soundly rejected at the $1 \%$ level of significance suggesting that the CPI basket contains highly heterogeneous components. Therefore, we conclude that the prediction errors are caused by item-specific heterogeneity.

We find that, in the presence of item-specific heterogeneity, the probability that prices remain unchanged is higher than in the single-sector model. We document that, to approximate the survivor function of the shared frailty model, it is necessary to use a lower value for the hazard rate in the Calvo model. 
Table 1

Descriptive statistics

\begin{tabular}{lccccc}
\hline \multirow{2}{*}{ Category } & \multirow{2}{*}{ total } & \multicolumn{4}{c}{ Per price spell } \\
\cline { 3 - 6 } & & mean & min & median & $\max$ \\
\hline Number of items & 498 & & & & \\
Number of price spells & 677169 & & & & \\
(First) entry time & & 0 & 0 & 0 & 0 \\
(Final) exit time & & 2.721 & 1 & 1 & 71 \\
Time at risk & 1798327 & 2.656 & 1 & 1 & 71 \\
Number of uncensored spells & 656581 & 0.970 & 0 & 1 & 1 \\
\hline
\end{tabular}

Source. - Retail price data used for the calculation of the Japanese CPI (2000-2005). 
Table 2

The Comparison of Calvo Model and Shared Frailty Model

\begin{tabular}{cccccc}
\hline & Hazard rate & \multicolumn{4}{c}{ Duration of price spells } \\
\cline { 3 - 6 } & of price & Mean & $25 \%$ & Median & $75 \%$ \\
& change & & & & \\
\hline Calvo (1983) model & 0.37 & 2.74 & 3.60 & 1.90 & 0.72 \\
Shared frailty model & - & - & 9.36 & 2.87 & 0.72 \\
\hline
\end{tabular}

Source. - Retail price data used for calculation of the Japanese CPI (2000-2005).

Note. - Hazard rate of price change is unweighted predicted hazard function. Price spell durations are reported in months. 
Figure 1

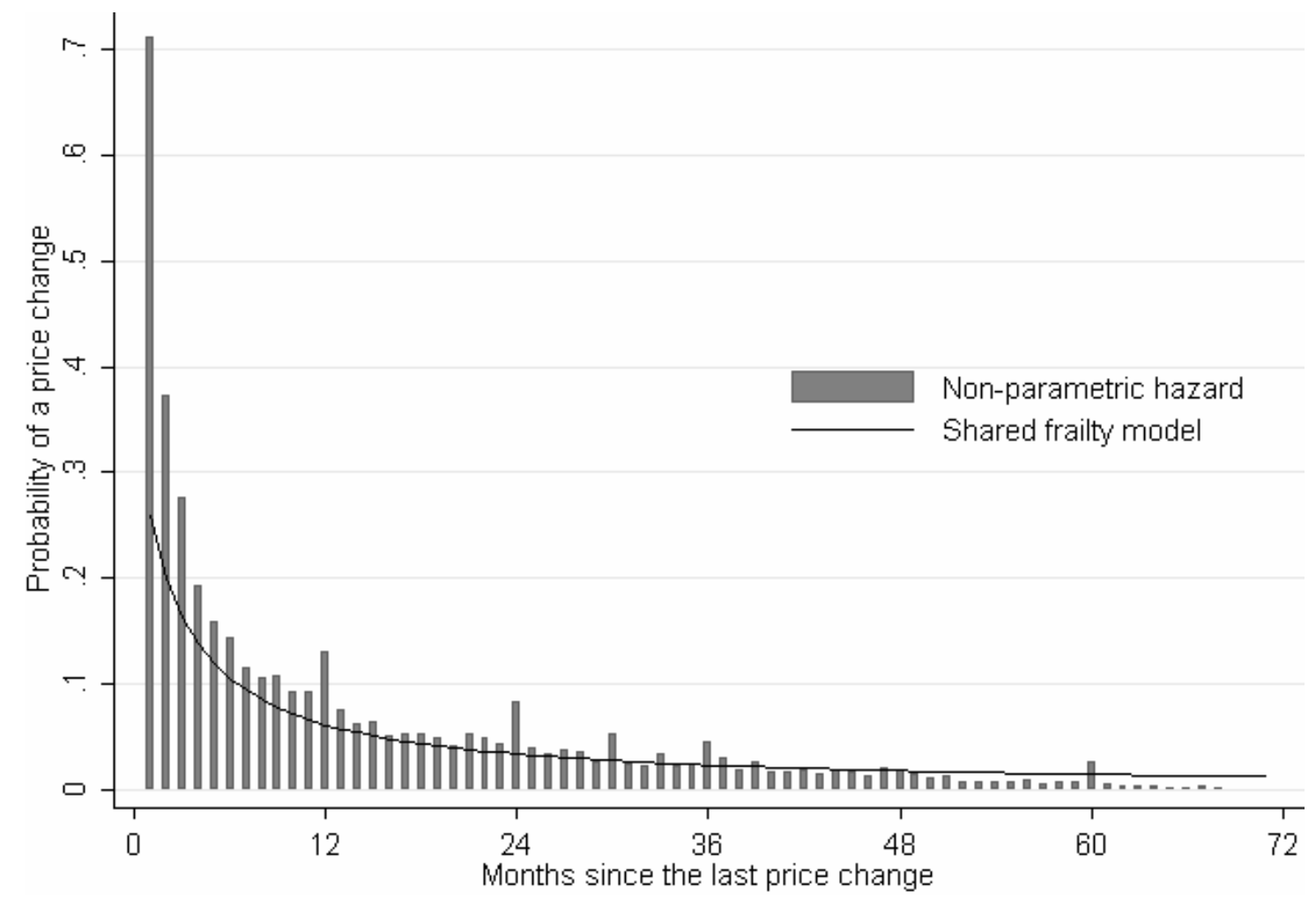

Population hazard from the exponential model with Gamma shared frailty vs. Kaplan-Meier estimator: Retail price data in Japan from 2000-2005: Unweighted sample (498 items). 
Figure 2

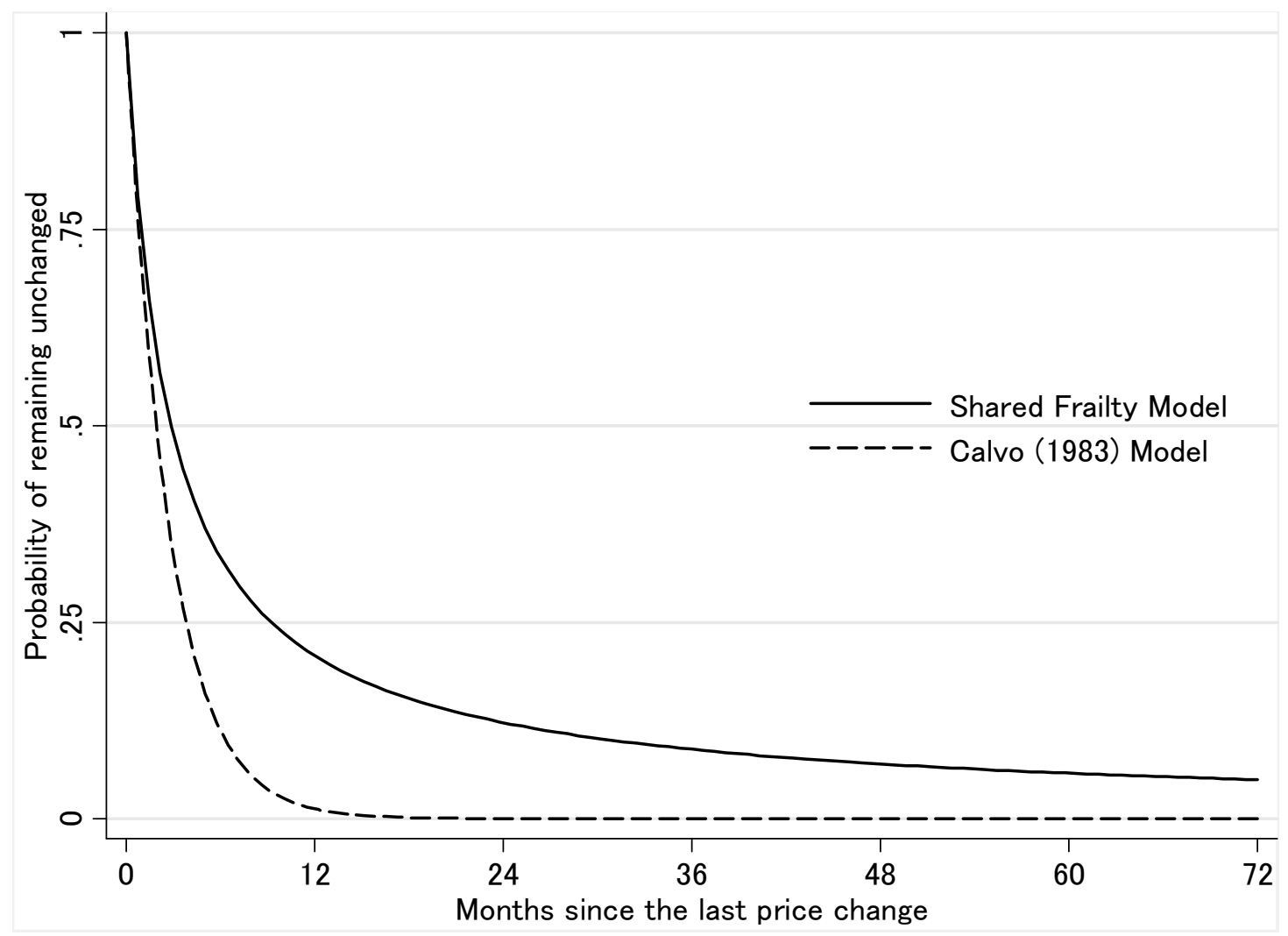

Survivor function from the shared frailty model and the Calvo (1983) model. Same data as Figure 1. 
Appendix A. The derivation of the joint survivor function for the $i$ th item.

From equation (7), we obtain

$$
\begin{aligned}
S\left(t_{i 1}, \cdots, t_{i n_{i}}\right) & =E_{v}\left\{\exp \left[-v \sum_{j=1}^{n_{i}} \Lambda\left(t_{i j} \mid X_{i j}\right)\right]\right\} \\
& =\int_{0}^{\infty} \exp \left[-v \sum_{j=1}^{n_{i}} \Lambda\left(t_{i j} \mid X_{i j}\right)\right] \frac{v^{(1 / \delta-1)} \exp (-v / \delta)}{\Gamma(1 / \delta) \delta^{1 / \delta}} d v \\
& =\frac{1}{\Gamma(1 / \delta) \delta^{1 / \delta}} \int_{0}^{\infty} \exp \left\{-v\left[\frac{1}{\delta}+\sum_{j=1}^{n_{i}} \Lambda\left(t_{i j} \mid X_{i j}\right)\right]\right\} v^{(1 / \delta-1)} d v .
\end{aligned}
$$

Letting $\frac{1}{\delta}+\sum_{j=1}^{n_{i}} \Lambda\left(t_{i j} \mid X_{i j}\right)=M$, we obtain

$$
S\left(t_{i 1}, \cdots, t_{i n_{i}}\right)=\frac{1}{\Gamma(1 / \delta) \delta^{1 / \delta}} \int_{0}^{\infty} \exp (-M v) \frac{\{M v\}^{(1 / \delta-1)}}{M^{(1 / \delta-1)}} d v .
$$

Define $y=M v$, so that $d v=M^{-1} d y$ and

$$
\begin{aligned}
S\left(t_{i 1}, \cdots, t_{i n_{i}}\right) & =\frac{1}{\Gamma(1 / \delta) \delta^{1 / \delta}} \int_{0}^{\infty} \exp (-y) \cdot y^{1 / \delta-1} \cdot M^{-1 / \delta+1} \cdot M^{-1} d y \\
& \left.=[\delta M]^{-1 / \delta} \quad \text { (By definition, } \Gamma(1 / \delta)=\int_{0}^{\infty} \exp (-y) \cdot y^{1 / \delta-1} d y\right) \\
& =\left[1+\delta \sum_{j=1}^{n_{i}} \Lambda\left(t_{i j} \mid X_{i j}\right)\right]^{-1 / \delta} .
\end{aligned}
$$

\section{Appendix B. The likelihood function for the $i$ th item}

Given the $i$ th heterogeneity $v_{i}$, the contribution to the likelihood for the $j$ th price spell in $i$ th item is

$$
\begin{aligned}
L_{i j}\left(v_{i}\right) & =S_{i j}\left(t_{i j}\right) \cdot \lambda_{i j}\left(t_{i j}\right)^{d_{i j}} \\
& =S\left(t_{i j} \mid X_{i j}\right)^{v_{i}} \cdot\left[v_{i} \lambda\left(t_{i j} \mid X_{i j}\right)\right]^{d_{i j}} \quad \text { (by equations (3) and (5)) }
\end{aligned}
$$

Using the expression of cumulative hazard function, equation (B.1) can be written as 


$$
L_{i j}\left(v_{i}\right)=\exp \left[-\sum_{j=1}^{n_{i}} \Lambda\left(t_{i j} \mid X_{i j}\right) \cdot v_{i}\right] \cdot\left[v_{i} \lambda\left(t_{i j} \mid X_{i j}\right)\right]^{d_{i j}} .
$$

Consequently, the conditional likelihood function for the $i$ th item is

$$
\begin{aligned}
L_{i}\left(v_{i}\right) & =\prod_{j=1}^{n_{i}} S\left(t_{i j} \mid X_{i j}\right)^{v_{i}} \cdot\left[v_{i} \lambda\left(t_{i j} \mid X_{i j}\right)\right]^{d_{i j}} \\
& =v_{i}^{D_{i}} \cdot \prod_{j=1}^{n_{i}} \exp \left[-\sum_{j=1}^{n_{i}} \Lambda\left(t_{i j} \mid X_{i j}\right) \cdot v_{i}\right] \lambda\left(t_{i j} \mid X_{i j}\right)^{d_{i j}},
\end{aligned}
$$

where $D_{i}=\sum_{j=1}^{n_{i}} d_{i j}$. Integrating out $v_{i}$, we obtain the unconditional likelihood

$$
L_{i}=\int_{0}^{\infty} L_{i}\left(v_{i}\right) g\left(v_{i}\right) d v_{i}
$$

where $g\left(v_{i}\right)$ is the density function given in equation (6). Therefore we have

$$
\begin{aligned}
L_{i} & =\int_{0}^{\infty} v_{i}^{D_{i}} \prod_{j=1}^{n_{i}} \exp \left[-\sum_{j=1}^{n_{i}} \Lambda\left(t_{i j} \mid X_{i j}\right) \cdot v_{i}\right] \cdot \lambda\left(t_{i j} \mid X_{i j}\right)^{d_{i j}} \cdot \frac{v_{i}^{(1 / \delta-1)} \exp \left(-v_{i} / \delta\right)}{\Gamma(1 / \delta) \delta^{1 / \delta}} d v_{i} \\
& =\left[\prod_{j=1}^{n_{i}} \lambda\left(t_{i j} \mid X_{i j}\right)^{d_{i j}}\right] \frac{1}{\Gamma(1 / \delta) \delta^{1 / \delta}} \int_{0}^{\infty} v_{i}^{\left(D_{i}+1 / \delta-1\right)} \prod_{j=1}^{n_{i}} \exp \left[-v_{i}\left(\frac{1}{\delta}+\sum_{j=1}^{n_{i}} \Lambda\left(t_{i j} \mid X_{i j}\right)\right)\right] d v_{i} .
\end{aligned}
$$

As in Appendix A, the integration in the last equality becomes

$$
\Gamma\left(1 / \delta+D_{i}\right)\left[\frac{1}{\delta}+\sum_{j=1}^{n_{i}} \Lambda\left(t_{i j} \mid X_{i j}\right)\right]^{-\left(D_{i}+1\right)} .
$$

Substituting (B.5) into (B.4), we recover equation (8). 


\section{References}

Álvarez, Luis J., Pablo Burriel, and Ignatio Hernando, 2005, Do Decreasing Hazard Functions for Price Changes Make Any Sense? ECB Working Paper Series No. 461.

Amemiya, Takeshi, 1984, Advanced Econometrics. (The MIT Press, Cambridge).

Aucremanne, Luc, and Emmanuel Dhyne, 2004, How frequently do prices change? Evidence based on the micro data underlying the Belgian CPI, ECB Working Paper Series No. 331.

Bils, M. and P. J. Klenow, 2004, Some evidence on the importance of sticky prices, Journal of Political Economy 112, 947-85.

Calvo, G., 1983, Staggered prices in a utility-maximizing framework, Journal of Monetary Economics 12 (3), 383-398.

Carvalho, Carlos, 2006, Heterogeneity in Price Stickiness and the Real Effects of Monetary Shocks, The B.E. Journal of Macroeconomics Vol. 2.

Cameron, A. Colin, and Pravin K. Trivedi, 2005, Microeconometrics: Methods and Applications. (Cambridge University Press, Cambridge).

Dhyne, E. et al., 2005, Price setting in the Euro area: some stylized facts from individual consumer price data, European Central Bank Working Paper Series 524.

Klein, J. P. and M. L. Moeschberger, 1997, Survival analysis- techniques for censored and truncated data. (Springer, New York).

Mosler, Karl, 2003, Mixture models in econometric duration analysis, Applied Stocastic Models in Business and Industry 19, 91-104.

Nakamura, Emi and Jón Steinsson, 2006a, Monetary Non-Neutrality in a Multi-Sector Menu Cost Models, mimeo available at http://www.people.fas.harvard.edu/ steinss/ hu-papers.html.

Nakamura, Emi and Jón Steinsson, 2006b, Five facts about prices: A Reevaluation of Menu Cost Models, mimeo available at http://www.people.fas.harvard.edu/ nakamura/papers.html..

Saita, Y, I. Takagawa, K. Nishizaki, and M. Higo, 2006, Kouribukka Toukei wo 
mochita Kakaku Nenchakusei no Keisoku (Price-setting in Japan: Evidence from CPI Micro Data), Bank of Japan Working Paper Series No.06-J-02 (in Japanese).

Yu, Binbing, 2006, Estimation of shared Gamma frailty models by a modified EM algorithm, Computational Statistics \& Data Analysis 50, 463-474. 